\title{
Do Time-Asymmetric Laws call for Time-Asymmetric Spacetime Structure?
}

\author{
Daniel Peterson \\ South Georgia State College \\ BIBLID [0873-626X (2017) 44; pp. 75-98]
}

\begin{abstract}
Many philosophers of physics take the failure of the laws of physics to be invariant under the time reversal transformation to give us good reason to think that spacetime is temporally anisotropic, yet the details of this inference are rarely made explicit. I discuss two reasonable ways of filling in the details of this inference, the first of which utilizes a symmetry principle proposed by John Earman and the second of which utilizes Harvey Brown's account of spacetime. I contend that neither of the resulting arguments is sound.
\end{abstract}

\section{Keywords}

Philosophy of physics, spacetime metaphysics, direction of time, symmetries, laws of nature

\section{Introduction}

Some philosophers, most notably Horwich (1987), Arntzenius (2004), and Maudlin (2007), appeal to temporally irreversible laws to justify their claim that there is a temporal anisotropy in the structure of spacetime itself. ${ }^{1}$ For instance, Arntzenius begins his paper as follows:

In this paper, I wish to consider the question whether the structure of spacetime includes a temporal orientation. Along with many others, I think that one will have compelling reasons to infer the existence of such a structure if one accepts local dynamical laws that are non-

${ }^{1}$ Exactly what Maudlin and Horwich in particular mean by a temporal anisotropy in spacetime is slightly ambiguous. In what follows, I take them to posit what I call a geometrical interpretation of this temporal orientation rather than a material one, which seems the most natural interpretation of their positions.

Disputatio, Vol. IX, No. 44, May 2017

Received: 30/07/2016 Revised: 16/11/2016 Accepted: 07/02/2017 
invariant under time-reversal. (Arntzenius 2004: 31)

Inferences like Arntzenius's from temporally irreversible laws to temporally anisotropic spacetime structure are typically quick, and it is often unclear what justifies them. Take, for instance, the following passage from Maudlin:

To begin with, the laws of physics as we have them....are not Time Reversal Invariant. The discovery that physical processes are not, in any sense, indifferent to the direction of time is important and well known: it is the discovery of the violation of so-called CP invariance, as observed in the decay of the neutral $\mathrm{K}$ meson. These decays are not invariant if one changes a right-handed for a left-handed spatial orientation (parity) and changes positive to negative charge (charge conjugation). According to the CPT theorem, any plausible quantum theory will be invariant under parity-plus-charge-conjugation-plus-time-reversal, so the violation of $\mathrm{CP}$ implies a violation of $\mathrm{T}$. In short, the fundamental laws of physics, as we have them, do require a temporal orientation on the spacetime manifold. (Maudlin 2007: 117, original emphasis)

In this passage, Maudlin moves from the fact that some physical processes are not time reversal invariant, which is supported by CP violation, to the claim that the kind of temporal spacetime orientation that he argues for in the rest of his chapter exists, and a similar passage can be found in Horwich (1987: 55).

I claim that, contrary to what these authors suggest, we have no good reason to infer the existence of temporal anisotropy in spacetime from only the temporally irreversible laws we currently believe fundamentally govern our world. In this paper, I draw on work by John Earman and Harvey Brown respectively to generate two seemingly reasonable justifications for this inference, then argue that both justifications fail. In the second section of the paper, I lay out a promising argument for temporal spacetime anisotropy from temporally irreversible physical laws. In the third section, I appeal to an argument from Earman (1989) to support the claim that nomic temporal irreversibility should lead us to introduce some temporally asymmetric structure to our best physical theories. In the fourth section, however, I argue that such a temporal asymmetry should not be interpreted as a fundamental feature of spacetime in our best physical theories, at least at the moment, and is better understood 
as a feature of either a law of nature or matter fields. In the fifth section, I consider an alternative argument that relies on the work of Brown (2005). Brown's view of spacetime both poses a challenge to my analysis in the fifth section and itself grounds an inference from temporally irreversible laws to temporally anistropic spacetime structure. However, I argue that Brown's view of spacetime is explanatorily inferior to an alternative view proposed by Janssen (2009) and, as such, should be rejected.

\section{An Argument for Temporal Asymmetry in Spacetime}

In the previous section I claimed that Arntzenius's and Maudlin's inferences were too quick. It seems that they need a premise along the lines of the following for their conclusion to follow: "If the fundamental physical laws are not symmetric under the time reversal transformation, then the spacetime required by these fundamental physical laws is not symmetric under the time reversal transformation." To be more explicit about the work done by this premise, I recast the inference as the following argument

\section{The Argument for Temporal Asymmetry in Spacetime}

(1) Not all of our best candidates for fundamental laws of physics are invariant under the dynamical symmetry ${ }^{2}$ time reversal.

(2) Any dynamical asymmetry of a physical theory gives us sufficient reason to posit the existence of some similarly asymmetric physical structure.

(3) Asymmetric structures posited because of a dynamical asymmetry in the fundamental laws are best interpreted as features of spacetime.

(4) The spacetime we are entitled to stipulate based on our best candidates for fundamental physical laws is temporally anisotropic. (from 1-3) here.

2 I am following Earman (1989) in my use of the term "dynamical symmetry" 
Experimental work on $\mathrm{K}$ and $\mathrm{B}$ meson decay ${ }^{3}$ gives us good reason to believe the first premise of the argument, so it is the second two premises of the above argument that draw our attention. Breaking the inference from a dynamical asymmetry to an asymmetry in spacetime into two separate premises makes it clear exactly what steps we must take when moving from facts about the laws to facts about spacetime. First, premise two claims that when we discover a dynamical asymmetry in the laws, we should posit the existence of some structure to explain this symmetry-breaking. Note that this asymmetric structure need not be some new structure. For instance, our laws may not be invariant under the 100-dimensional group of rotations mathematically represented by $\mathrm{SO}(100)$, but we need not posit some new structure to explain this fact if our theory already appeals to structures like tensors that fail to be invariant under such transformations. However, if we find that the laws fail to be invariant under some transformation under which we take all of our currently posited physical structures to be invariant, the second premise of the argument licenses us to either add some new structure that breaks this symmetry or replace an existing structure with a new structure that breaks the symmetry.

But just because we have posited some asymmetric structure to explain an asymmetry in the dynamical laws does not mean that we know how to interpret the object we have posited. For instance, we can explain the failure of laws to be parity reversal invariant by following a suggestion from Pooley (2003) that there is a field that determines the parity or "handedness" of every physical process. The laws, we can claim, fail to be parity reversal invariant because they refer to this parity field, so we have an explanation for the fact that the laws are not parity reversal invariant but do not yet know how we should understand this parity field. If, as the third premise of the Argument for Temporal Asymmetry in Spacetime suggests, we should understand this parity field as an element of spacetime structure,

\footnotetext{
${ }^{3}$ See Sachs (1987), chapter 9 for a detailed discussion of K meson decay and Abe et. al. (2001) for a discussion of B meson decay. When coupled with the CPT theorem, the $\mathrm{CP}$-invariance of $\mathrm{K}$ and $\mathrm{B}$ meson decay gives us good indirect evidence for failures of time reversal invariance in quantum field theory, and recent experimental results from the BABAR research group at SLAC provide direct evidence of T-reversal violations in $\mathrm{B}^{0}$ meson systems (Lees et. al. 2012).
} 
then we have a parity analogue for the Argument for Temporal Asymmetry in Spacetime. But we may resist this move and claim instead that we should posit another of Pooley's candidates to break the parity symmetry, namely a law that claims that all processes of a certain type have the same orientation. If we can posit a law instead of some other structure to explain the breaking of parity reversal symmetry, then we have a challenge to premise 3 .

But even if we find reasonable grounds to exclude such a law from our theory, we may choose to interpret the field Pooley proposes as a material field within spacetime instead of as an element of spacetime itself. More specifically, there are two different interpretations of what Pooley's proposed field represents that will be important for our purposes. The first takes this field to be a feature of spacetime or to represent features of spacetime itself. The second interpretation takes this field to be a mathematical description of a feature of matter fields, like a spatial arrow with which certain kinds of matter come equipped, much like the electric field in classical electromagnetism. This second interpretation takes Pooley's field to be a map from points in spacetime to structural features of spacetime even though what the field itself represents is not an element of the spacetime structure. Our first interpretation takes the field to encode geometric features of spacetime while our second interpretation takes the field to encode features of the contents of spacetime. I will call any interpretation, like the first above, which takes an object to encode geometric features of spacetime "geometrical", and I will call any interpretation, like the second above, which takes an object to encode features (even geometric features) of the contents of spacetime "material". Any object interpreted geometrically I will call a "geometrical object", ${ }^{4}$ and any object interpreted materially I will call a "material object". Keep these distinctions in mind as I will rely on this terminology particularly in section 4.

Thus, even if we can justify premise 2 , we are faced with the

${ }^{4}$ Not to be confused with a geometric object — many geometric objects may be interpreted either geometrically or materially. Saying that an object is geometric tells us about the mathematical role it plays in a theory while saying that an object is geometrical tells us how to interpret that particular object. I have bolded the term "geometrical" throughout this paper to help avoid confusing it with the term "geometric". 
question of both what object to posit to break the symmetry and how to interpret that object. One needs an argument for premise 3 that justifies interpreting the structure posited as a result of premise 2 as an element of spacetime if one wants to make the inference from dynamical asymmetries to asymmeries of spacetime. Before moving on to this second project, however, I will briefly consider the status of premise 2 .

\section{Earman's SP2}

Let us excise one objection to premise 2 briefly mentioned above before we get started. One reasonable way to account for the failure of the laws to fail to be time reversal invariant is to posit some conventional, non-local law. As an analogue of Pooley's parity law, we could treat "all physically possible temporally ordered sequences of events have the same temporal orientation" as an additional fundamental law of our best theories. Assuming that our spacetime is temporally orientable, we then need only conventionally stipulate at some point in spacetime which direction is "past" and which direction is "future" to fix the temporal orientation at all times. Such a law should not be interpreted as a feature of spacetime, so it provides us with sufficient grounds to reject the second premise of the Argument for Temporal Asymmetry in Spacetime. There are those who might object to this law because it is non-local, so, in fairness to the proponent of the Argument for Temporal Asymmetry in Spacetime, I will grant, for the sake of argument, that such laws don not give us good reason to reject premise 2 .

If we reject conventional, non-local laws as a reasonable way to explain temporal asymmetries, there are many ways that one could justify the second premise of the Argument for Temporal Asymmetry in Spacetime. Consider the following principle:

(SP2) All spacetime symmetries of a theory $\mathrm{T}$ are dynamical symmetries of $\mathrm{T}$.

This symmetry principle is proposed and supported by Earman (1989), and its contrapositive seems a good candidate to provide a link between claims about temporally irreversible laws and temporal spacetime asymmetries: if we have some diffeomorphism defined on 
our spacetime manifold that fails to be a dynamical symmetry of our theory (that is, if we have an asymmetry in the laws), then SP2 tells us that that same diffeomorphism should fail to be a spacetime symmetry of our theory (that is, we should presumably have an asymmetry in an object representing the structure of spacetime) as well.

To understand SP2, we must start from Earman's definitions of dynamical and spacetime symmetries. Earman sets up his account of symmetries as follows: Let $\mathrm{T}$ be a theory whose models are of the form $<M, A_{1}, A_{2}, \ldots, P_{1}, P_{2}, \ldots>$, where $M$ is a differentiable manifold; $A_{i}$ are the "absolute objects"; and $P_{i}$, the "dynamical objects", are the geometric-object fields that characterize the physical contents of the manifold. Earman does not provide us with a formal definition for his absolute objects, but he endorses an account shared by Friedman (1983), who does provide such a definition. Friedman defines absolute objects as geometric-object fields that satisfy the following condition:

A geometric object $\Phi_{\mathrm{i}}$ is an absolute object of a theory T just in case for any two models $<M, \Phi_{1}, \ldots, \Phi_{n}>$ and $<M, \Psi_{1}, \ldots, \Psi_{n}>$ of $T$, for every $p \in M$, there are neighborhoods $A, B$ of $p$ and a transformation $\mathrm{h}: \mathrm{A} \rightarrow \mathrm{B}$, such that $\Psi_{\mathrm{i}}=\mathrm{h} \Phi_{\mathrm{i}}$ on $\mathrm{A} \cap \mathrm{B}$.

Earman's account provides us with a fairly clear sense of how to construct a theory's models. For instance, a physical theory in Aristotelian spacetime has models with absolute objects like a field picking out a preferred spatial origin and with dynamical objects such as fields picking out the spatiotemporal locations of physical objects, electromagnetic fields, etc.

Earman then defines spacetime and dynamical symmetries as follows:

Any diffeomorphism $\Phi: M \rightarrow M$ such that $\Phi * A_{i}=A_{i}$ for all $i$ is a spacetime symmetry of the model $<\mathrm{M}, \mathrm{A}_{1}, \mathrm{~A}_{2}, \ldots, \mathrm{P}_{1}, \mathrm{P}_{2}, \ldots>$. A diffeomorphism $\Phi$ is a spacetime symmetry of a theory $T$ just in case it is a spacetime symmetry of all of T's models.

A diffeomorphism $\Phi: M \rightarrow M$ is a dynamical symmetry of a theory $T$ iff $\Phi * \mathrm{~A}_{\mathrm{i}}=\mathrm{A}_{\mathrm{i}}$ and $\Phi * \mathrm{P}_{\mathrm{i}}=\mathrm{P}^{\prime}{ }_{\mathrm{i}}$ for all $\mathrm{i}$, where $<\mathrm{M}, \mathrm{A}_{1}, \mathrm{~A}_{2}, \ldots, \mathrm{P}_{1}, \mathrm{P}_{2}, \ldots>$ and $<\mathrm{M}, \mathrm{A}_{1}, \mathrm{~A}_{2}, \ldots, \mathrm{P}_{1}^{\prime}, \mathrm{P}_{2}^{\prime}, \ldots>$ are both models of a theory $\mathrm{T}$. 
A spacetime symmetry is a spacetime diffeomorphism that leaves the absolute objects unaffected, and a dynamical symmetry is a spacetime symmetry that transforms the dynamical objects in such a way that the result of the transformation still obeys the fundamental dynamical laws. In Earman's terms, we might support the second and third premises of the Argument for Temporal Asymmetry in Spacetime as follows: SP2 implies that, if we have a dynamical asymmetry, then we have a spacetime asymmetry, meaning at least one absolute object in some model of our theory is not mapped to itself by the diffeomorphism in question. But if any asymmetry in the absolute objects is best understood as an asymmetric feature of spacetime itself, SP2 allows us to take the third premise for granted and skip right to the conclusion.

Earman's SP2 is a reasonable symmetry principle, but there are limits to how far it can get us. In order to establish that SP2 supports premise 3 generally, one needs to argue that absolute objects must be interpreted as geometrical objects. This may not seem a hard argument to make. After all, Earman (1989) takes absolute objects to represent the "fixed space-time structure" (45). But given Friedman's definition, it is easy to see that being an absolute object is neither necessary nor sufficient for being interpreted as a feature of spacetime itself. For instance, take any non-vanishing vector field, such as the Newtonian gravitational field or the electromagnetic field. It follows from the vector straightening theorem and Friedman's definition that any such vector field constitutes an absolute object, yet we clearly would not want to interpret all non-vanishing vector fields as geometrical objects. ${ }^{5} \mathrm{~A}$ similar result can be established for any constant scalar field. Friedman also points out that, while the metric tensor is an absolute object in special relativity, it is not so in general relativity; since the metric tensor provides us with important information about the structure of spacetime in general relativistic theories, being an absolute object clearly is not necessary for representing a geometrical object either. The question of whether or not the

\footnotetext{
${ }^{5}$ Philosophers following Brown (2005) might not be too concerned about the consequence that all non-vanishing vector fields represent elements of spacetime structure given their interpretation of spacetime, but Brown's view poses other problems I will discuss later in this paper.
} 
mathematical object that breaks the time reversal invariance of the dynamical laws is an absolute object in our physical theories is thus separate from the question of how we should interpret that object. SP2 gives us reason to accept the second but not the third premise of the Argument for Temporal Asymmetry in Spacetime, so the third premise of this argument must find its support elsewhere.

\section{An object to break the symmetry}

Premise 3 requires us to consider what a reasonable temporally asymmetric structure to posit in light of premise 2 might be. The following structure serves as a temporally asymmetric physical object that can account for temporally irreversible laws. Take the orientation field represented by $\mathrm{F}$ to be a map from spacetime points to light cones $;^{6} \Phi$ allows us, at each point in spacetime, to pick out a single unique, consistent light cone which we take to be the future light cone at $p .{ }^{7}$ We are then faced with the following problem regarding $\Phi$ : should we interpret $\Phi$ as a geometrical or material object? In other words, should we think of a temporal orientation field like the metric tensor or like an electric field? ${ }^{8}$

One first intuition might be that theoretical virtues can help settle the question, and one virtue in particular, methodological conservatism, seems promising. The conservative principle best suited to our project licenses the inference in the following passage from Sklar:

\footnotetext{
${ }^{6} \Phi$ is clearly not the only object that could break the symmetry, nor do we need to add some new object to our theory to break the symmetry if the theory in question already fails to be time reversal invariant. Still, what I say about $\Phi$ will apply equally well to any object, novel or not, that reveals a theory's failure to be symmetric under the time reversal transformation.

${ }^{7}$ The notion of consistency I have in mind here comes from Earman (1974). Consistency requires that there is no point $\mathrm{x}$ in spacetime such that continuous transport of the light cone around a closed loop based at $\mathrm{x}$ causes points previously on one side of the light cone to now fall in the other side of the light cone. Also, note that a field $\Phi^{\prime}$ that picks out the past light cone at each point in spacetime would work just as well as $\Phi$ for our purposes.

${ }^{8}$ Note that, even interpreted materially, the spacetime on which $\Phi$ is defined must be temporally orientable, but that this alone does not show that the spacetime on which $\Phi$ is defined is temporally oriented.
} 
We have to choose among alternative hypotheses all of which seem equally warranted on the basis of reasonable inference from the evidential data. But suppose one of the hypotheses is "more in conformity with preexisting theory" — more "like it in structure," say, or more "conceptually continuous" with it. Then that hypothesis is preferred. (Sklar 1975: 31)

This principle tells us to prefer the hypothesis that deviates as little from our previously-held hypotheses as possible, all other things being equal. We can apply this principle to the geometrical/material debate as follows: though we may not have a clear account of how to determine which objects are geometrical and which are material, most authors agree about many of the features that we should interpret as geometrical according to particular physical theories. The metric tensor and the properties we can determine from it such as spacetime curvature, the connection, light cone structures, and the spacetime separation are understood geometrically, not materially, in our best currently available spacetime theories. Thus, the methodologically conservative among us will want our new geometrical objects to stick as close to these original geometrical objects as possible. Let us then provisionally state that we should take the geometrical objects to be the ones that we can derive from the metric tensor alone. We may posit new sorts of geometrical objects if empirical data or other theoretical virtues drive us to do so, but, all other things being equal, it is better to stick with theories that take as geometrical only the sorts of objects we are used to taking as geometrical.

Why should we be so resistant to changes in geometrical objects? Our best theories of spacetime physics are our best theories for a reason, namely because they are empirically successful and have been for a while. Scientific realists typically take the empirical success of a scientific theory to provide a (potentially defeasible) reason to believe that the scientific theory has gotten certain features of the world right. We may no longer believe that Newtonian mechanics is literally true, but we do believe that it got a number of important things right about the relationship among, for instance, the mass, velocity, acceleration, and position of medium-sized physical objects. Likewise, we may think that our current set of geometrical objects posited by our best available physical theories is incomplete because, 
like all physical theories, it will likely be modified or discarded in the future; however, since we are not in a position to know what it is that our current set of geometrical objects gets right, the best policy would be to stick to this set as closely as possible while still allowing these objects to play the role in our physical theories that they currently play.

Having identified the metric and what follows from it as our litmus test for identifying geometrical objects, we can now apply our conservative principle to help us determine when we should identify a particular object as geometrical or material. The metric tensor is symmetric in both space and time; that is, for any metric tensor $g_{a b}$, it follows that $g_{\mathrm{ab}}(\mathbf{v}, \mathbf{w})=g_{\mathrm{ab}}(\mathbf{w}, \mathbf{v})=$ for any vectors $\mathbf{v}, \mathbf{w}$ in the tangent space. If the metric alone does not tell us whether, for instance, some point $\mathrm{p}$ is in the past or future of another point $\mathrm{q}$, then we need to rely on some sort of supplemental structure to provide this information. We have two options: we can treat this asymmetrical structure as a geometrical or material object. If we treat it as a geometrical object, however, we risk violating Sklar's conservative principle. After all, relativistic metric tensors do not provide us with any information about preferred directions in space or time; why should we expect the metric tensor found in our new theory to play such a different role?

So methodological conservatism may give us reason to think that any orientation field like the one I have proposed is best treated as a material rather than a geometrical object because it performs a task separate from the kinds of tasks performed by the metric tensor in our best available physical theories. However, this reason is not decisive. Other considerations may lead us to believe, for methodologically conservative reasons, that interpreting $\Phi$ materially is incorrect since it has many features (not carry energy, providing only a direction but not a magnitude, etc.) that many other material fields do not. Thus, methodological conservatism alone may not be sufficient to resolve this puzzle unless we wish to table-thumping about intuitions.

Let us take a different tack: what it is we want spacetime to do for us in our physical and metaphysical theories? Consider the case of special relativity. What was it that motivated physicists to posit the existence of spacetime as opposed to simply remarking on the fact 
that all of the laws seemed to be Lorentz-invariant? The concept of spacetime is valuable because it provides a unifying explanation for a diverse range of phenomena that we may think cry out for explanation. Spacetime serves as a kind of meta-law, explaining and justifying the Lorentz-invariance of physical laws instead of requiring that we take the Lorentz-invariance of these laws as a brute fact. So, if all the laws seem to be Lorentz-invariant, then we have a fact about these laws to explain, and geometrical objects may be posited to explain this fact.

But imagine that we are given a case like Tooley's (1977) garden where we find that one particular region of spacetime is "special" in some sense. Perhaps, for instance, we have a physical theory that fails to be spatial translation invariant because the laws allow some phenomena in a region $\mathrm{X}$ that are denied outside of $\mathrm{X}$. Should we posit that the region privileged by this strange law is somehow privileged by the spacetime structure of our world? Well, it depends on what facts we need to explain. If all of our other physical theories are spatial translation invariant, then it seems the only fact we have to explain is the failure of one particular natural law to be invariant under spatial translation. We may explain this fact by tracing the failure of spatial translation invariance back to initial conditions that fail to be spatial translation invariant despite spatial translation invariant micro-laws, or we may posit some object that fails to be spatial translation invariant that some laws couple to. But to posit that this preferred region $\mathrm{X}$ is a special geometrical object is to leave more unexplained than it ever explained in the first place. If spacetime itself fails to be spatial translation invariant, then why are so many of our other laws spatial translation invariant? If we were to investigate and find that, in every case, laws that fail to be spatial translation invariant were more empirically adequate than their spatial translation invariant alternatives, positing some spacetime structure that failed to be spatial translation invariant would not be explanatorily embarrassing, but that is not the case in our hypothetical. So it seems like the explanatory gains outweigh the costs when we posit an asymmetry in spacetime to explain an asymmetry that most or all dynamical laws seem to exhibit; however, if most of the laws are invariant under some symmetry and we have only a few laws that are not, then positing an asymmetric feature of spacetime to explain these few 
asymmetries comes at a great explanatory cost, and the benefits are not worth it.

Put differently, I am suggesting that we adopt the following general rule, a variant of inference to the best explanation:

(EXPL): We have more reason to assign interpretation $I$ to mathematical object $o$ than we have reason to assign interpretation $I^{\prime}$ to $o$ only if $I$ provides us with a better explanation for the physical phenomena that motivated our positing $o$ in the first place than $I^{\prime}$ provides.

There may be difficult cases where reasonable individuals disagree over which theory or interpretation is truly more explanatory, but in many situations, our paradigm cases of excellent explanations (e.g. those that are more unifying, those that are less ad hoc, etc.) will provide us with an acceptable metric by which to judge which theory or interpretation is more explanatory and will frequently lead to general agreement. EXPL does not tell us that the more explanatory theory or interpretation is true, nor does it suggest that we ignore or dismiss theories or interpretations that we do not believe at this time. It does suggest, however, that the currently available evidence should lead us to believe the theory or interpretation that provides us with better explanations and fewer unexplained phenomena than its rivals.

EXPL suggests that we should only interpret $\Phi$ as geometrical if all or most of the phenomena accounted for by our theory fundamentally fail to be time reversal invariant. If many (but not all) of the fundamental laws of our theory, for instance, are time reversal invariant, then a geometrical $\Phi$ would account for some laws but not others, and we would need an explanation for this strange feature of spacetime. A material $\Phi$, on the other hand, would not have so difficult a burden as it could be interpreted as merely describing features of the matter governed by the temporally irreversible laws. The Tviolations that lead us to believe that the fundamental laws of physics are not all time reversal invariant have only been observed in two different types of mesons. We have no reason, at present, to believe that the symmetry-breaking is more widespread since T-violations have not been observed in or inferred from experiments involving numerous other particle collisions. T-violating phenomena, then, 
seem comparatively rare and exotic. Even if we take the standard model alone as our best available physical theory, the vast majority of the terms in the Lagrangian we would write down to describe all of the particles in the universe would be time reversal invariant. If we posit, then, that there is a particular field, $\Phi$, that some mesons couple to and that other particles do not, we have an explanation (or at least the beginning of an explanation) for the failure of time reversal invariance we observe. If we posit that spacetime itself fails to be time reversal invariant, however, we must explain why the Lorentz-invariance of spacetime makes every term in the Lagrangian Lorentz-invariant while the failure of spacetime to be time reversal invariant does not actually imply that many (or most) of the terms in the Lagrangian for the universe fail to be time reversal invariant.

For those who remain unconvinced, let us consider an analogy with the electric field. When faced with phenomena suggesting the presence of an electric field, physicists could have chosen to interpret this field geometrically or materially but chose to interpret it materially. Presumably, this is at least in part due to the fact that not all material objects interact with the electric field in interesting ways. Uncharged particles are not directly affected by such a field, so the electric field does not seem suitably universal to be interpreted geometrically. This example may lead to a new interpretive principle that even those wary of EXPL should be willing to adopt:

(UNIV): Geometrical objects help us explain a wider (or more universal) array of physical phenomena than material objects, so an object should only be interpreted geometrically if the physical phenomena that object is needed to explain are sufficiently numerous.

The term "sufficiently numerous" may be somewhat vague, but we can use past cases as interpretive guides here. When we consider the salient universality standards used to apply this principle to the case of the material electric field, it seems hard to see how such standards could consistently support interpreting $\Phi$ as a geometrical object. Thus, considerations of other materially interpreted fields suggest that it is better to interpret $\Phi$ materially than geometrically.

One might reasonably worry that, in looking for general principles like EXPL and UNIV to tell us how to interpret $\Phi$, we are 
adopting the wrong philosophical methodology. In particular, one might look to Arntzenius (1997) as a model for the right way to approach questions about how to properly interpret temporal asymmetries. In his paper, Arntenzius argues that claims about how to properly interpret temporal asymmetries should be resolved by carefully considering differing interpretations of the theory in which the asymmetry appears. In particular, Arntzenius argues that collapse theorists and Bohmians should understand the temporal asymmetry in quantum mechanics differently. Similarly, then, we might worry that my project, which relies on general explanatory principles rather than specific interpretations of the underlying physical theories, is incapable of properly justifying my claim that, given the current state of our best physical theories, we have good reason to interpret $\Phi$ materially rather than geometrically.

I certainly grant that considering different interpretations of quantum field theory may provide us with some illumination on the question of how to interpret $\Phi$. However, it is worth noting that Arntzenius uses these interpretations to adjudicate between interpreting the temporal asymmetry of quantum mechanics as picking out a preferred temporal direction in spacetime and between interpreting the temporal asymmetry of quantum mechanics as a constraint on the initial conditions of the universe. Arntzenius, in his analysis, does not explicitly consider the question of whether or not the preferred temporal direction in spacetime, which he associates with collapse theories, ought to be interpreted geometrically or materially, which is the central question under consideration here; that is, he is fundamentally concerned with the question of whether or not a temporal arrow exists, not the question of how, if such an arrow exists, it ought to be interpreted. Consider how Arntzenius motivates his analysis of quantum mechanics using the toy case of robots moving around a plane. Arntzenius presents two possible explanations for our discovery that all of the robots take the same steps when they happen to be in the same location: we can infer that some arrow is associated with each location, or we can infer that each robot has some sort of internal program that tells it how to move based on its last steps. But the question of whether the spatial arrow associated with the first option should be understood geometrically (e.g. positing that the path traversed by each robot follows a geodesic 
based on the geometry of the plane) or materially (e.g. positing that there is a constant wind that always blows robots at the same point in the same direction) is never discussed. So, while Arntzenius has given us good reason to believe that some questions about how to interpret temporal asymmetries may be answered by appealing to different interpretations of the specific theory in which the temporal asymmetry appears, his analysis has not shown itself to be capable of answering the specific question considered here, nor does it invalidate my use of general interpretive principles like EXPL and UNIV to justify my position.

Treating $\Phi$ as a material object leaves us with less to explain than treating it as a geometrical object does and better aligns with our treatment of fields like the electric field in the past, so we have a good reason to think that $\Phi$ is a material rather than geometrical object; however, should we find that other physical theories are improved by setting them in spacetimes containing a geometrical object like $\Phi$, then we should adopt a different conclusion. Without such physical theories to appeal to, however, we seem to have a good reason to reject the third premise of the Argument for Temporal Asymmetry in Spacetime by taking $\Phi$ to be material, and so we have a good reason to believe the Argument for Temporal Asymmetry in Spacetime unsound.

\section{But what is spacetime?}

In the previous section, I covertly endorsed what I take to be a fairly intuitive view of spacetime shared by Balashov and Janssen (2003) and Janssen (2009), namely that the spacetime of a physical theory is more fundamental than its dynamical laws. Consider, for instance, Coulomb's law. This law allows us to determine the motion of charged particles in classical electromagnetism, and the influence of the electromagnetic force is proportional to the inverse square of the distance between the particle and the origin of the force. It is the fundamental spacetime relations that tell us the distance between the particle and the force's origin. The dynamical laws rely (both explanatorily and ontologically) on spacetime in that facts about distances, for instance, determine the form certain laws take. The basic picture of Balashov and Janssen's account is of an underlying, unify- 
ing spacetime that grounds facts about the dynamical laws and allows us to explain the behavior and symmetries of various laws by appealing to the structure of spacetime.

There is, however, an alternative picture available, one which denies my move in the previous section and provides independent support for the last two premises of the Argument for Temporal Asymmetry in Spacetime. Brown (2005) and Brown and Pooley (2006) suggest that it is the laws of physics that ground, justify, and explain our claims about spacetime, not the other way around. ${ }^{9}$ For instance, the laws of physics are not Lorentz invariant because spacetime itself is Lorentz invariant; rather, physicsts claim that spacetime is Lorentz invariant as a way of capturing an important fact about our dynamical laws, namely their Lorentz-invariance. Spacetime is, according to Brown and Pooley, a "glorious non-entity", incapable of explaining facts about the dynamical laws in the way that Janssen suggests. It seems fitting to think of Brown's account as taking a similar view towards spacetime as the advocate of Humean supervenience takes towards natural laws: just as the Humean claims that natural laws are no more than generalized regularities that satisfy some important cognitive criteria, Brown claims that spacetime is no more than a mathematical representation of certain features many of the laws seem to share..$^{10}$ It is the laws that explain and justify facts about this spacetime structure, not the other way around. Relying on a causal account of explanation, Brown suggests that Minkowski spacetime

\footnotetext{
${ }^{9}$ The fact that Brown makes claims about ontological as well as explanatory priority is noted by Norton (2008). I should also note here that there is some ambiguity over the correct way to read Brown's claims. For the purposes of this paper, I take Brown to be making a supervenience claim about the ontological priority of laws over spacetime structure rather than adopting a geometric conventionalist stance. If one accepts the latter reading, then the inference at the heart of this paper follows trivially, and the Argument for Temporal Asymmetry in Spacetime is unnecessary. Note, however, that if Brown's argument (regardless of how one reads him) is supposed to hinge on his claim that all genuine explanation is causal explanation, then the response to Brown-centered arguments for temporal spacetime asymmetries that I provide in this section will hold regardless of how one understands Brown's position.
}

${ }^{10}$ A discussion of Brown's account in Frisch (2011: 128) makes these similarities quite striking. 
does no real explanatory work: it cannot cause matter to move in certain ways, so how can we take it to explain why matter moves as it does? Given the explanatory deficiencies of spacetime, Brown suggests we do the parsimonious thing and remove spacetime, insofar as it represents something separate and apart from a codification of facts about the physical laws, from our ontology.

If Brown is correct, then not only is there no real, meaningful geometrical/material distinction, but there is also a fairly straightforward argument for asymmetries in spacetime from dynamical asymmetries; if there is nothing more to spacetime than what is already encoded in the facts about the dynamical laws, then finding out that the dynamical laws are temporally asymmetric should lead us to believe that the spacetime we posit on their account is likewise temporally asymmetric. In order to head off objections to the view I have laid out in the previous sections, I must provide compelling reasons to reject Brown's account.

Brown and Janssen seem to take the best arguments in favor of their respective positions to lie in the explanatory features of their favored accounts, so let us begin there. Brown argues that there is a gap in explanations that rely on spacetime to explain, for instance, why moving rods contract or why light rays follow geodesics. The problem, Brown says, is that spacetime cannot provide causal explanations of material phenomena, so it cannot explain them at all. If we want to explain why a law is Lorentz-invariant, for instance, Brown suggests that an adequate explanation would be to derive this law from a more fundamental Lorentz-invariant law. If the law in question is fundamental, however, then Brown does not provide us with any resources to explain the law's Lorentz-invariance. Regardless of the law's status, it would be unacceptable, Brown claims, to try to explain the Lorentz-invariance of a law by appealing to the structure of spacetime as an independent entity.

Janssen, however, argues that, without spacetime, there is a particularly odd fact that Brown and his allies need to account for. Special relativity is a Lorentz-invariant theory, and treatments of other theories such as the electroweak theory and quantum chromodynamics assume Lorentz-invariance from the start. The laws of our best available physical theories all seem to invariant under the same set of symmetries, which seems to call out for an explanation. 
Without some unifying explanation, it seems quite the coincidence that all of our best available laws just happen to share this feature in common. ${ }^{11}$

Brown can appeal to an underlying physical theory invariant under the symmetries that require explanation here. Perhaps many of the laws we use are Lorentz-invariant because the fundamental physical theory that underlies them is also Lorentz-invariant. But what if there is not one single dynamical physical theory that underwrites all of the laws in question? If a single underlying physical theory exists, then Brown may have the upper hand since brute facts about that theory seem no worse off explanatorily than brute facts about spacetime. But if pluralism in physics wins the day and no empirically adequate single unifying theory is ever found, Brown's account faces Janssen's worry anew since he cannot explain why all of the more fundamental laws are invariant under the symmetries they are invariant under. Because the single unified theory response considered here relies on the existence of a theory not currently available to us, I think it is reasonable to discount this "one fundamental theory" response until such a theory is discovered. So, absent a unifying theory of everything for Brown to hang his argument on, the best line of argument available to Brown in response to Janssen is to argue that it is acceptable to take some facts about laws as brute.

Both camps can dig in their heels here: Brown can claim that the fact that all of the best fundamental laws seem to be Lorentz-invariant is not the sort of fact that calls out for explanation (or may even be the sort of fact that can never be explained) while Janssen can claim that the fact that moving rods contract is explained (insofar as it can be explained) by the nature of spacetime but that not all explanations need be causal explanations. The current state of the debate between these two views on spacetime seems to rest on the reasonableness of these two explanatory claims.

Brown claims that all genuine explanations are causal, yet there seem to be good reasons to think that there are genuine instances of non-causal explanation. Brown's position implies that "A explains $\mathrm{B}$ " is true just in case there is a causal relationship between A and B such that A (or a substructure of A) causes B (or a substructure of

${ }^{11}$ This point is made by Frisch (2011: 182) as well. 
B), but there are counterexamples that seem to suggest otherwise. For instance, we may explain a drop in the pressure of an ideal gas by appealing to the gas's increasing volume, and we may explain an increase in an ideal gas's volume by appealing to a drop in the gas's pressure. Yet causal relationships are asymmetric since causes temporally precede their effects. So no matter what causal story we decide to tell about the ideal gas, one of our explanatory claims will be unaccounted for. Causal accounts of explanation are similarly poor at accounting for explanations of laws that appeal only to other laws or meta-laws. Unless we are willing to accept the claim that features of one law can cause features of another law, Brown may have a hard time explaining why he takes it to be the case that the Lorentz-invariance of law $\mathrm{L}$ can be explained by the Lorentz-invariance of the more fundamental law $\mathrm{L}^{\prime}$ from which $\mathrm{L}$ can be derived. The relationship here does seem to be explanatory, but, at least on most common accounts of causation, it does not seem to be distinctly causal. We have good reason, then, to believe that not all explanation is causal.

I bring these instances of non-causal explanation to the fore to set up what I take to be a better account of explanation than Brown advocates, namely one that relies on the more general notion of dependence. Jansson (2011) (not to be confused with Janssen) has advocated an account of explanation that runs essentially as follows: we can truthfully say "A explains B" just in case both A and B occur and, when $A$ occurs, $B$ also occurs in virtue of $A$, and when $A$ does not occur, B fails to occur in virtue of A's failure to occur. These dependency relationships help us capture instances of causal explanation but are more general than causal relationships, so they seem better suited to deal with my previous counterexamples that showed that causation was not necessary for explanation. On Jansson's account, the ideal gas law encodes a nomic dependency relation between the volume and pressure of an ideal gas, so the pressure of the gas can explain its volume and vice-versa even if there is no causal relation we can posit to justify both explanations. And features of the laws are certainly dependent on (if not caused by) features of more fundamental laws. We thus have good reason to prefer a more general account of explanation like the dependency account to Brown's more limited, "causal relationships only" account.

Armed with the dependency account of explanation, we may 
attempt to break the explanatory stalemate in the Brown/Janssen debate since we now seem to have good reason to think that spacetime provides us with a genuine explanation of phenomena like contracting rods. These phenomena depend on the structure of spacetime since moving rods contract in virtue of the Lorentz-invariance of spacetime and a relation between the contents of spacetime and spacetime relations that connect them to one another. If the structure of spacetime changes, we can expect this phenomenon to likewise change, so the sort of dependency relation Janssen might posit in defense of his view of spacetime seems to fit the explanatory criteria laid down by the dependency account of explanation. ${ }^{12}$ Likewise, spacetime can explain the fact that laws in theories like quantum chromodynamics and the electroweak theory are Lorentz-invariant. The relation between spacetime and these theories is such that we can determine the Lorentz-invariance of these theories given only a Lorentz-invariant spacetime and the relation between spacetime and the dynamical laws. If the structure of spacetime changes so that it is no longer Lorentz-invariant, the relation between spacetime and the laws requires a change in the Lorentz-invariance of these laws as well. Again, we have a genuine explanation of a phenomenon that requires us to appeal to spacetime.

But such explanatory considerations need not be all that leads us to prefer Janssen's account to Brown's. Consider counterfactual claims about the laws of nature, such as "Even if the standard model is false, the laws that govern particles in Lisbon are the same as the laws that govern particles in Tokyo". The truth of such claims can be understood straightforwardly in Janssen's account — since our spacetime is spatial translation invariant, even if we admit that the laws are not what we think they are, the true laws that govern particle behavior (whatever they are) must be spatial translation invariant if we are right about the kind of spacetime we live in. In other words, Janssen's account explains how facts about spacetime can ground the truth of certain counterfactual claims. Brown's account, on the

${ }^{12}$ Brown may object that Janssen needs to say a bit more about the relations that obtain between spacetime and material objects, and this may be desirable for a more satisfying explanation of special relativistic phenomena, but such details are not necessary for showing that spacetime explains phenomena in special relativity. 
other hand, seems incapable of explaining how such counterfactual claims could be true since the story of why we take our spacetime to be spatial translation dependent involves us generalizing symmetries from specific physical theories like the standard model itself. So, if robust explanations of certain counterfactual truths are a desideratum for our interpretation of spacetime, it seems we have another good reason to prefer Janssen's account to Brown's.

So where does this leave us? As I have shown, Janssen's spacetime, when coupled with a dependency account of explanation, is capable of explaining not only the phenomena Brown mentioned but also a phenomenon that Brown's account must take as brute: the fact that all of the best physical laws seem to be Lorentz-invariant. Likewise, Janssen's account provides a clear explanation for the truth of certain counterfactual claims about the laws and their relationship to spacetime. Thus, since Janssen's spacetime is capable of doing all the explanatory work Brown claimed it could not while also explaining phenomena that Brown's account is unable to explain, we have a compelling reason to favor Janssen's view of spacetime and a response to objectors who would rely on Brown's account to deny my geometrical/material distinction and justify the second and third premises of the Argument for Temporal Asymmetry in Spacetime.

\section{Conclusion}

I have attempted to support Arntzenius's, Horwich's, and Maudlin's inferences from temporal asymmetries in dynamical laws to temporal asymmetries in spacetime and argued that neither of the approaches I have examined is successful. The Argument for Temporal Asymmetry in Spacetime seems promising and could be supported by appeals to Earman's symmetry principle SP2 or Brown's account of spacetime. Earman's SP2 does not establish the existence of a symmetry-breaking object that is best interpreted as geometrical. I have argued that we have two reasonable ways of breaking the symmetry: we can posit a non-local, conventional dynamical law, or we can posit an object like $\Phi$ which, except in cases where failures of time reversal invariance in fundamental physical phenomena are widespread, we have a good reason to think should be understood materially instead of a geometrically. So, even if one accepts the 
second premise of the argument, the third premise is likely false, and the Argument for Temporal Asymmetry in Spacetime fails. While Brown's account of spacetime could indeed provide the Argument for Temporal Asymmetry in Spacetime with the support it requires, the view of spacetime suggested by Janssen provides us with more satisfying explanations for why the laws behave as they do while still providing explanations for the phenomena Brown charges it cannot explain. Thus, we should reject Brown's account, and once again we find the Argument for Temporal Asymmetry in Spacetime unsound. I do not take my conclusion here to be the final word on the matter but hope that my analysis will challenge those who casually move from talking about temporal asymmetries in the dynamical laws to talking about temporal asymmetries in spacetime itself to explicitly justify their inference and deal with the difficulties this inference presents. ${ }^{13}$

Daniel Peterson

South Georgia State College dan.james.peterson@gmail.com

\section{References}

Abe, K. et al. 2001. Observation of large CP violation in the neutral B meson system. Physical Review Letters 87.

Arntzenius, F. 1995. Indeterminism and the direction of time. Topoi 14: 67-81.

Arntzenius, F. 1997. Mirrors and the direction of time. Philosophy of Science 64: S213-S222.

Arntzenius, F. 2004. Time reversal operations, representations of the Lorentz group, and the direction of time. Studies in History and Philosophy of Science Part B 35: 31-43.

Balashov, Y. and Janssen, M. 2003. Presentism and relativity. The British Journal for the Philosophy of Science 54: 327-346.

Brown, H. 2005. Physical Relativity: Space-Time Structure From A Dynamical Perspective. Oxford: Oxford University Press.

Brown, H. and Pooley, O. 2006. Minkowski space-time: a glorious non- entity. In The Ontology of Spacetime. Cambridge: Elsevier.

Earman, J. 1974. An attempt to add a little direction to "the problem of the

${ }^{13}$ I am particularly grateful to David Baker, Gordon Belot, Sarah Moss, Laura Ruetsche, Chip Sebens, Larry Sklar, James Wells, and two anonymous referees for helpful comments and discussion on earlier drafts of the paper. 
direction of time". Philosophy of Science 41: 15-47.

Earman, J. 1989. World Enough and Space-Time: Absolute versus Relational Theories of Space and Time. Cambridge: MIT Press.

Friedman, M. 1983. Foundations of Space-Time Theories. Princeton: Princeton University Press.

Frisch, M. 2011. Principle or constructive relativity. Studies in History and Philosophy of Modern Physics 42: 176-183.

Horwich, P. 1987. Asymmetries in Time: Problems in the Philosophy of Science. Cambridge: MIT Press.

Janssen, M. 2009. Drawing the line between kinematics and dynamics in special relativity. Studies In History and Philosophy of Science Part B: Studies In History and Philosophy of Modern Physics 40: 26-52.

Jansson, L. 2011. Explanation and dependence. PhD dissertation. The University of Michigan.

Lees, J. et. al. 2012. Observation of time-reversal violation in the $\mathrm{B}^{0}$ meson system. Physical Review Letters 109(21).

Maudlin, T. 2007. The Metaphysics Within Physics. Oxford: Oxford University Press.

Norton, J. 2008. Why constructive relativity fails. The British Journal for the Philosophy of Science 59: 821-834.

Pooley, O. 2003. Handedness, parity violation, and the reality of space. In Symmetry in Physics: Philosophical Reflections. Cambridge: Cambridge University Press.

Sachs, R. 1987. The Physics of Time Reversal. Chicago: University of Chicago Press.

Sklar, L. 1975. Methodological conservatism. The Philosophical Review 84: 374-400.

Streater, R., and A. Wightman. 1980. PCT, Spin, Statistics, and All That. Princeton: Princeton University Press.

Tooley, M. 1977. The nature of laws. Canadian Journal of Philosophy 7: 667-698. 